\title{
A!
}

This is an electronic reprint of the original article.

This reprint may differ from the original in pagination and typographic detail.

Moerland, R. J.; Kuipers, L.; Kaivola, M.

\section{Emission control with metallic hole arrays}

Published in:

PLASMONICS: METALLIC NANOSTRUCTURES AND THEIR OPTICAL PROPERTIES IX

DOI:

$10.1117 / 12.893895$

Published: 01/01/2011

Document Version

Publisher's PDF, also known as Version of record

Please cite the original version:

Moerland, R. J., Kuipers, L., \& Kaivola, M. (2011). Emission control with metallic hole arrays. In MI. Stockman (Ed.), PLASMONICS: METALLIC NANOSTRUCTURES AND THEIR OPTICAL PROPERTIES IX [809635] (Proceedings of SPIE; Vol. 8096). SPIE. https://doi.org/10.1117/12.893895

This material is protected by copyright and other intellectual property rights, and duplication or sale of all or part of any of the repository collections is not permitted, except that material may be duplicated by you for your research use or educational purposes in electronic or print form. You must obtain permission for any other use. Electronic or print copies may not be offered, whether for sale or otherwise to anyone who is not an authorised user. 


\section{Emission control with metallic hole arrays}

R. J. Moerland, L. Kuipers, M. Kaivola

R. J. Moerland, L. Kuipers, M. Kaivola, "Emission control with metallic hole arrays," Proc. SPIE 8096, Plasmonics: Metallic Nanostructures and Their Optical Properties IX, 809635 (20 September 2011); doi: 10.1117/12.893895

SPIE Event: SPIE NanoScience + Engineering, 2011, San Diego, California, United States 


\title{
Emission control with metallic hole arrays
}

\author{
R.J. Moerland ${ }^{a}$, L. Kuipers ${ }^{b}$, M. Kaivola ${ }^{a}$ \\ ${ }^{a}$ Aalto University, School of Science, Department of Applied Physics, \\ P.O.Box 15100, FI-00076 Aalto, Finland \\ ${ }^{b}$ FOM Institute for Atomic and Molecular Physics (AMOLF), \\ Kruislaan 407, NL-1098SJ, Amsterdam, The Netherlands
}

\begin{abstract}
Adding holes in a periodic arrangement to metallic thin films greatly affects the optical properties of the metal film. The compound structure can exert a large influence on electromagnetic fields that interact with the hole array. Many parameters affect the actual response of the hole arrays to electromagnetic fields, such as the periodicity, the size of the holes and their shape. Here, we will show by calculation that the angular emission and lifetime of emitters, embedded in hole arrays comprised of rectangular holes with varying aspect ratio, depend strongly on the hole aspect ratio. Specifically, changing the aspect ratio of the holes leads to a large variety in far-field emission patterns and a more than 10-fold changes in decay rate of a single emitter placed in the central hole of such an array.
\end{abstract}

Keywords: plasmonics, hole array, angular emission, lifetime, single emitter

\section{INTRODUCTION}

Non-periodic metal surfaces and (nanoscale) metal objects can have a significant effect on the luminescence of emitters. ${ }^{1-15}$ Plasmon resonances of small metal particles have been shown to enhance the Raman signal from single adsorbed molecules, ${ }^{11,12}$ the fluorescence of organic dye molecules,${ }^{13-15}$ as well as the luminescence of silicon quantum dots ${ }^{16,17}$ and electroluminescence of devices based on silicon. ${ }^{18}$ The increased yield of radiative processes having a low quantum efficiency, together with a spectral selectivity that can be engineered, make that plasmon resonances in general have a high applicability for devices and sensors. In periodic structures, plasmon resonances can have counter-intuitive results. For example, hole arrays can exhibit an average transmission for specific wavelengths of light that greatly exceeds the expectation based on the transmission of a single hole. ${ }^{19}$ Additionally, it has been shown that, for various shapes and sizes, the transmission of hole arrays can be changed drastically, solely by a variation of the shape of the individual holes. ${ }^{20}$ Local resonances, or shape resonances, also play a role in the transmission of light through hole arrays ${ }^{21-23}$ and in the efficiency of non-linear processes in hole arrays. $^{24}$

Luminescence of emitters near and in hole arrays has been studied earlier: various interactions of emitters with hole array structures have been reported in literature, such as enhanced fluorescence by molecules, ${ }^{25,26}$ plasmon excitation in hole arrays by molecules ${ }^{27}$ and a reduction in the lifetime of molecules. ${ }^{28,29}$ However, the specific role of hole shape on fluorescence has only gotten minimal attention. Here, we will show by calculation that the angular emission and lifetime of emitters, embedded in hole arrays comprised of rectangular holes with varying aspect ratio, depend strongly on the hole aspect ratio. The strong dependence of these properties on hole shape opens up new routes to control the emission of emitters.

Send correspondence to R. J. Moerland, robert.moerland@aalto.fi

Plasmonics: Metallic Nanostructures and Their Optical Properties IX, edited by Mark I. Stockman,

Proc. of SPIE Vol. 8096, 809635 - @ 2011 SPIE · CCC code: 0277-786X/11/\$18 · doi: 10.1117/12.893895

Proc. of SPIE Vol. 8096 809635-1 


\section{DIPOLAR EMITTERS IN INHOMOGENEOUS MEDIA}

Though the lifetime of an emitter is inherently a quantum-mechanical phenomenon, classical electrodynamics can be applied if an emitter is viewed as a classical dipole..$^{2,30-33}$ The decay rate of a single emitter can quite easily increase an order of magnitude in the vicinity of metal structures that support plasmonic modes, such as nanoscale spheroids made of gold or silver. ${ }^{13,14,17,33}$ Also near thin films of metal, a strong dependence of the decay rate of an emitter on the distance to the metal film can be found, a phenomenon explained by the excitation of SPP modes in the metal film by the emitter. ${ }^{2,33-35}$

The rate at which a molecule dissipates power is defined as the decay rate $\gamma$. The contributions to $\gamma$ can be subdivided into the following parts:

$$
\gamma=\gamma_{i}+\gamma_{n}+\gamma_{r}
$$

where $\gamma_{i}$ is the rate at which power is dissipated intra-molecularly, $\gamma_{r}$ is the rate at which power is dissipated into the far field and $\gamma_{n}$ is the rate at which power is dissipated non-radiatively, like certain surface plasmon polariton modes. The relative change in the lifetime of a molecule when placed in another environment is connected to the total decay rate and dissipated power as follows: ${ }^{31,32}$

$$
\tau_{0} / \tau=\gamma / \gamma_{0}=P / P_{0},
$$

where $\tau$ is the lifetime of the molecule in the environment and the subscript 0 denotes the value of $\tau, \gamma$ and $P$ in a reference environment, usually free space. The change in decay rate $\gamma / \gamma_{0}$ can be calculated with classical electrodynamics. ${ }^{2,30-33}$ If a single Hertzian dipole, as an approximation for a luminescing emitter, is considered, then $\gamma / \gamma_{0}$ is related to the dipole moment and local electric field as

$$
\frac{\gamma}{\gamma_{0}}=\frac{P}{P_{0}}=\frac{\Im\left\{\mathbf{p}^{*} \cdot \mathbf{E}_{1}\right\}}{\Im\left\{\mathbf{p}^{*} \cdot \mathbf{E}_{\mathbf{l}_{0}}\right\}} .
$$

Here, $\mathbf{p}$ is the dipole moment of the source and $\mathbf{E}_{l}$ the value of the electric field at the location of the dipole. Due to the complexity of the geometry and materials, analytical solutions to Maxwell's equations are difficult, if not impossible, to obtain. We therefore pursue similar methods as published earlier, ${ }^{7,8,10,36,37}$ where the change in decay rate $\gamma / \gamma_{0}$ is numerically estimated by solving Maxwell's equations in a finite element version of the original. In order to calculate the angular emission patterns, tangential field components, obtained close to the truncation of the calculation volume, are stored for each time step. Using this information, the far-field radiation power per unit solid angle $P(\theta, \phi)$ is obtained by Fourier-transforming these near fields into far fields according to methods published elsewhere. ${ }^{38}$

\section{SIMULATIONS}

\subsection{Model set-up}

The total radiated power by a classical dipole in a hole array was calculated with models of hole arrays samples that are smaller than arrays typically used in experiments. The origin of the truncation stems from the fact that a typical hole array sample is too large when modeled numerically to fit inside the working memory of a desktop computer. Here, the number of holes had to be limited due to computer memory and time limitations. However, since experimental evidence is available which shows that arrays of $9 \times 9$ holes behave essentially the same as arrays of $19 \times 19$ holes, ${ }^{39}$ it is assumed that the reduction of the number of holes does not considerably change the local environment of emitters present in the central hole.

The model hole arrays consist of a dielectric substrate with $\varepsilon=2.25$, on which a layer was placed of $200 \mathrm{~nm}$ thickness of a material that has an electric permittivity that follows a Drude model. The Drude model's parameters were chosen such that the electric permittivity of the material describes that of gold in the range of $350-400 \mathrm{THz}(\approx 750-850 \mathrm{~nm})$. In this layer, 81 rectangular holes in a $9 \times 9$ arrangement were placed. The electric permittivity of the holes was set to that of vacuum. The models were truncated just beyond the area of the holes and an absorbing boundary layer was applied. ${ }^{40}$ The periodicity of the holes was fixed to $410 \mathrm{~nm}$ in both the $x$ - and $y$-direction. The area of an individual hole is fixed to $34 \cdot 10^{3} \mathrm{~nm}^{2}$, but the aspect ratio $\Delta x / \Delta y$ 


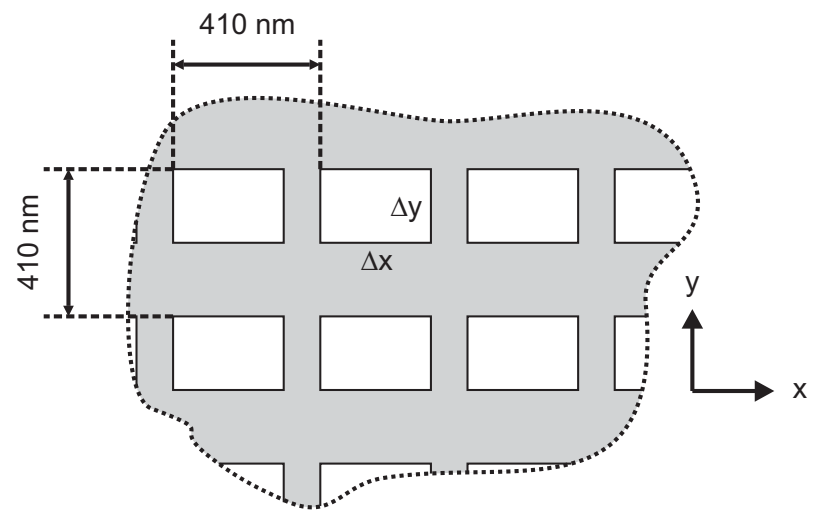

(a)

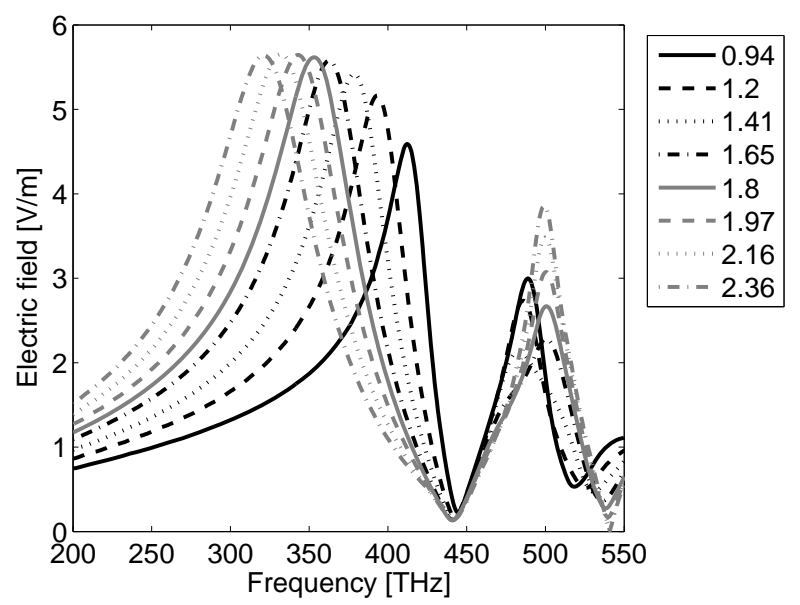

(b)

Figure 1: (a) Schematic layout of a part of the model of a hole array used for calculations. Only a small portion of the $9 \times 9$ holes is shown. The period of the holes is $410 \mathrm{~nm}$ in both the $x$ - and $y$-direction. The area of the holes is fixed to $34 \cdot 10^{3} \mathrm{~nm}^{2}$, but the aspect ratio $\Delta x / \Delta y$ is varied between 1 and 2.36.

(b) The results of a calculation of the electric field amplitude at the center of a hole in an infinite hole array, where a $y$-polarized plane wave was used as excitation. The amplitude of the excitation was $1 \mathrm{~V} / \mathrm{m}$. As the hole aspect ratio increases, the strongest resonance shifts to lower frequencies (longer wavelengths), whereas the location of the second, slightly weaker, resonance is more or less fixed at $\approx 500 \mathrm{THz}(600 \mathrm{~nm})$.

was varied from 1 to 2.36 . Here, $\Delta x$ is the size of a hole in the $x$-direction and $\Delta y$ is the size of a hole in the $y$-direction. See also Fig. 1a.

Employing periodic boundary conditions, the electric field strength at the center of a hole of an effectively infinite array of holes was calculated for a $y$-polarized plane wave. The plane wave's propagation direction was perpendicular to the metal surface and its amplitude was $1 \mathrm{~V} / \mathrm{m}$. Fig. $1 \mathrm{~b}$ displays the result of the calculation, for various aspect ratios. Clearly visible is that two resonances occur in the structure. As the hole aspect ratio increases, the strongest resonance shifts to lower frequencies (longer wavelengths), whereas the location of the second, slightly weaker, resonance is more or less fixed at $\approx 500 \mathrm{THz}(600 \mathrm{~nm})$. In the next sections, we focus on the behavior of a single emitter which emits in the frequency range of the strongest resonance.

\subsection{Lifetime of emitters versus aspect ratio}

For each aspect ratio, calculations were carried out for two orientations of a dipole in the central hole. The dipole was positioned at the center of the central hole, $10 \mathrm{~nm}$ away in the $z$-direction from the dielectric substrate. For each aspect ratio, $P / P_{0}=\gamma / \gamma_{0}$ was calculated for dipoles with $x$ - and $y$-orientations. Here, $P_{0}$ is the power dissipated by a dipole in free space. Due to the time-consuming nature of the calculations, $z$-oriented dipoles were not regarded. The results of the calculations are shown in Fig. 2. For an $x$-oriented dipole, the relative decay rate is maximal when the dipole is in a hole array with an aspect ratio of 0.94 . As the aspect ratio increases, the decay rate of a dipole with $x$-orientation drops, for all frequencies included in the calculation. The maximum decay rate occurs at $400 \mathrm{THz}$, regardless the aspect ratio. In contrast, for $y$-oriented dipoles, the calculated decay rates show a maximum that is a function of frequency and aspect ratio. The maximum decay rate shifts down to lower frequencies as the aspect ratio increases. From these simulations the conclusion can be drawn that hole arrays are highly dispersive devices: the decay rates of embedded emitters is highly sensitive to the emission frequency and orientation of the emitters in the hole array. 


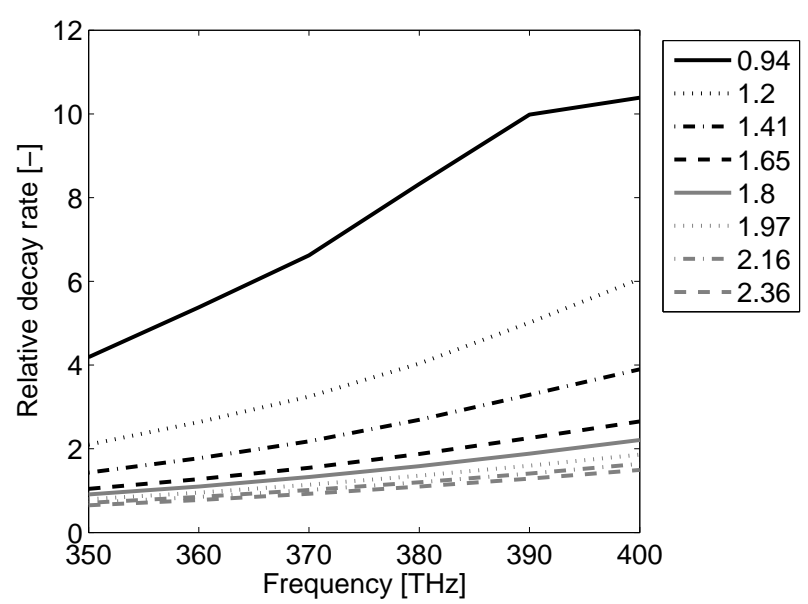

(a) $x$-oriented dipole

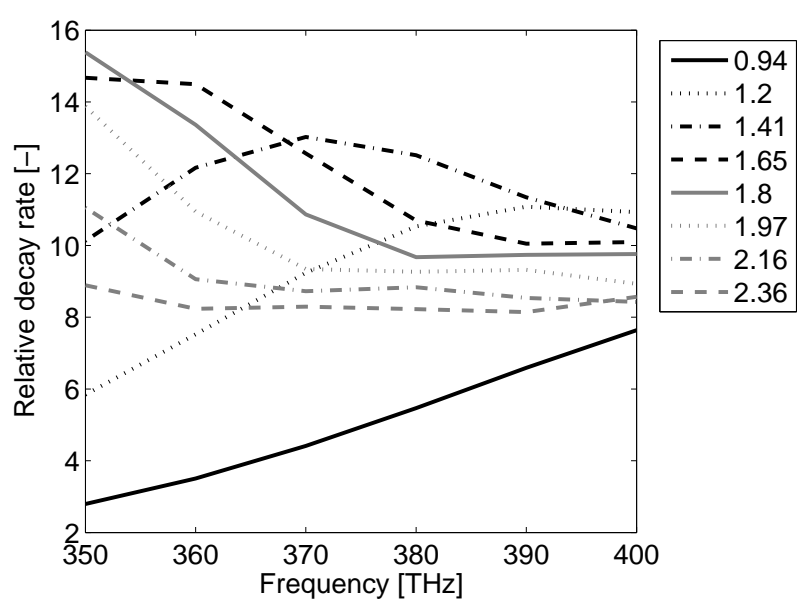

(b) $y$-oriented dipole

Figure 2: Simulated decay rate of dipoles located at the center of the central hole in a $9 \times 9$ hole array. In (a), the results of the simulation for $x$-oriented dipoles is displayed. The relative decay rate is the highest for an aspect ratio of 0.94 . As the aspect ratio increases, the relative decay rate drops for all frequencies included in the calculation. For $y$-oriented dipoles, for which the results are displayed in (b), there is a clear maximum in the relative decay rate which depends on the frequency and aspect ratio of the holes. With increasing aspect ratio, the maximum in relative decay rate shifts to lower frequencies.

To compare the found decay rates with an in-plane dipole $10 \mathrm{~nm}$ above bare glass, instead of free space, the numbers found must be divided by 1.3. The factor 1.3 accounts for the increase in decay rate due to coupling of the near field of an in-plane dipole to a glass substrate.

\subsection{Angular emission patterns versus aspect ratio}

Emission patterns as a function of aspect ratio were calculated at a single frequency of $375 \mathrm{THz}(800 \mathrm{~nm})$ for a single $y$-oriented dipole situated at the center of the central hole. The frequency at which the calculation was performed is chosen such that the peak in field strength (Fig. 1b) and decay rate (Fig 2b) moves across this particular frequency and reveals the influence of solely the aspect ratio on the emission pattern. The results of this calculation are shown in Fig. 3b-h as a function of $\theta$ and $\phi$, where $\theta$ and $\phi$ are defined as shown in Fig. 3a. As the aspect ratio increases, an emission lobe develops at the center of the emission pattern. A further increase of the aspect ratio causes two side lobes to emerge which are more intense than the center peak. Fig. 3 shows that the radiation pattern of a single $y$-oriented dipole, at the center of the central hole, strongly depends on the aspect ratio. It can therefore be expected that emitters at different locations and with different orientations have an emission pattern that strongly depends on the aspect ratio as well.

Beside the angular emission pattern as a function of aspect ratio, the angular emission of a single $y$-oriented dipole in a hole array with a fixed aspect ratio of 1.65 was calculated as a function of frequency. The results of this calculation are displayed in Fig. 4a-f. The properties of the hole arrays depend strongly on frequency, which results in a highly modified emission pattern when the emission frequency of the $y$-oriented dipole is increased from 350 to $400 \mathrm{THz}$. As the frequency increases, the magnitude of the center lobe drops and two side lobes emerge.

\section{CONCLUSIONS}

Through simulations, hole arrays are shown to be highly dispersive: the decay rate and emission pattern of a single dipole in a hole array is strongly dependent on the emission frequency and its orientation. For emitters with moderate to low intrinsic quantum efficiency, the higher decay rates due to coupling to plasmonic modes - which 


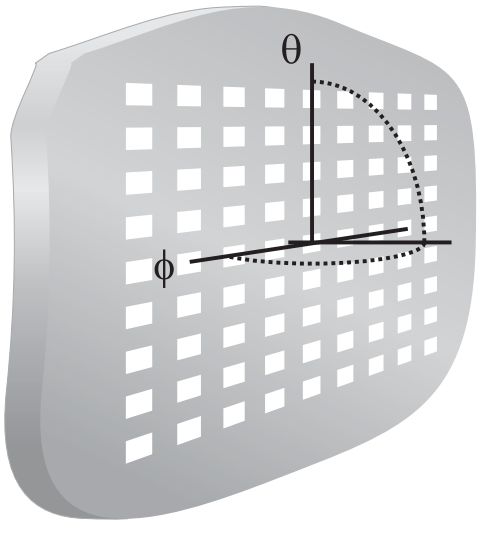

(a)

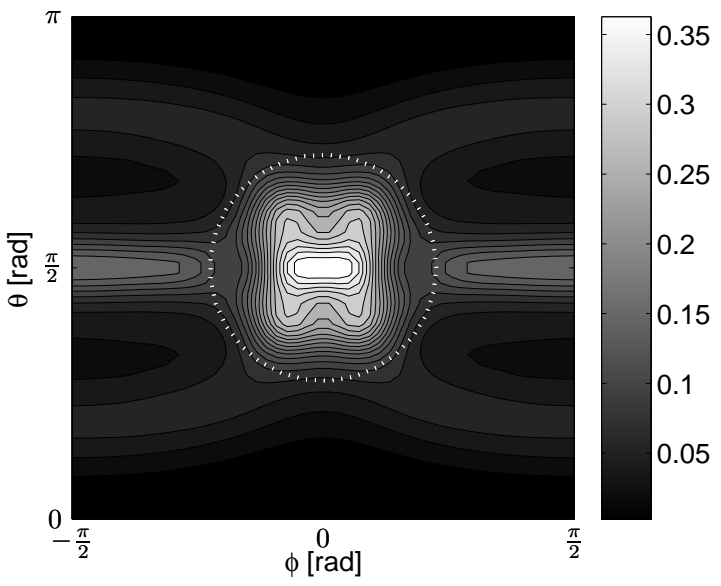

(c) 1.41

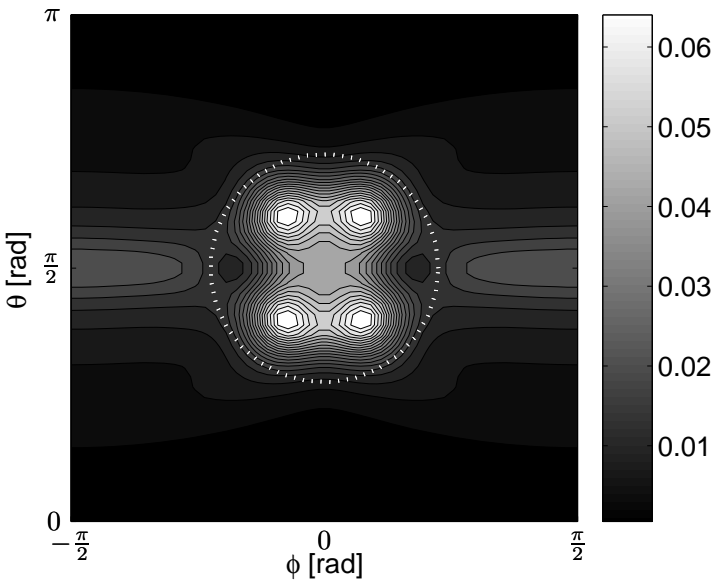

(b) 0.94

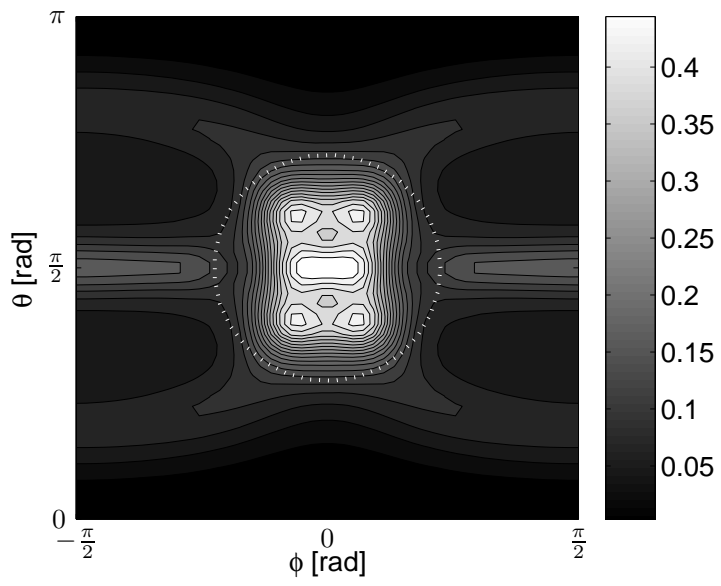

(d) 1.65

Figure 3: Angular emission pattern of a single $y$-oriented dipole at the center of the central hole in a $9 \times 9$ hole array. (a) Definition of $\theta$ and $\phi$. (b)-(h) Patterns were calculated at a frequency of $375 \mathrm{THz}(800 \mathrm{~nm})$. Subscripts underneath each figure give the aspect ratio of the hole array. A white dotted circle depicts the collection cone of an air objective with an NA of 0.65. As the aspect ratio increases, an emission lobe develops at the center of the emission pattern. With a further increase of the aspect ratio, two side lobes emerge which are more intense than the center peak. 


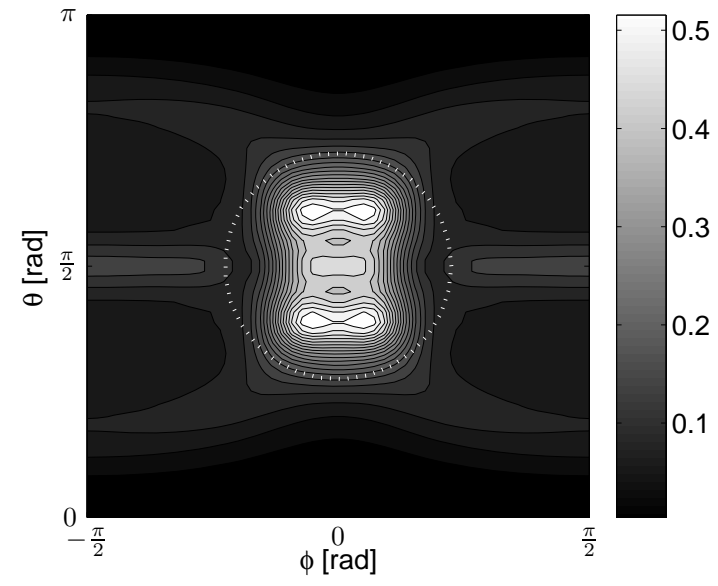

(e) 1.8

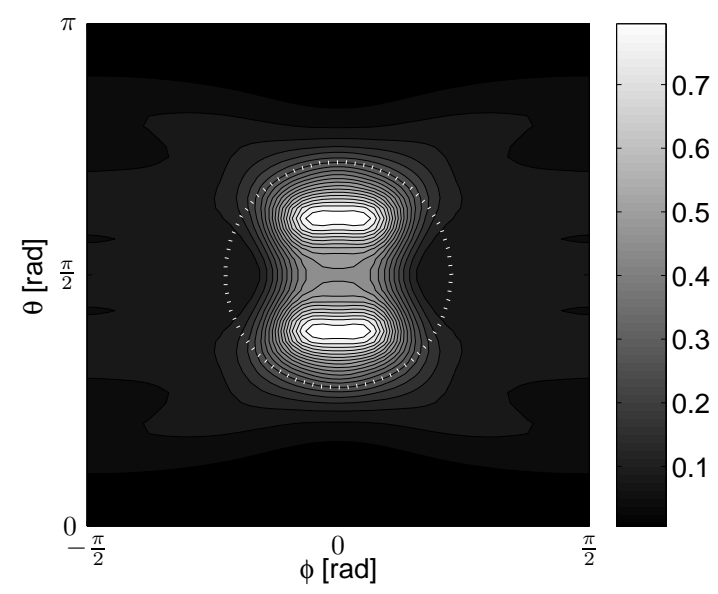

(g) 2.16

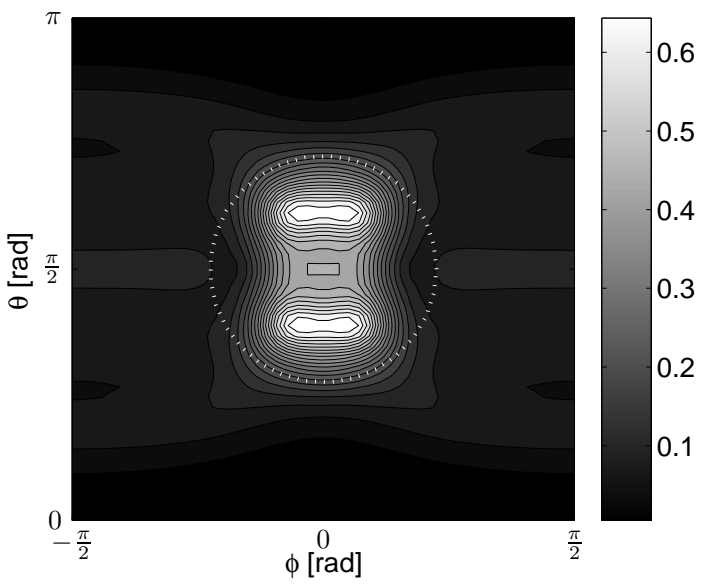

(f) 1.97

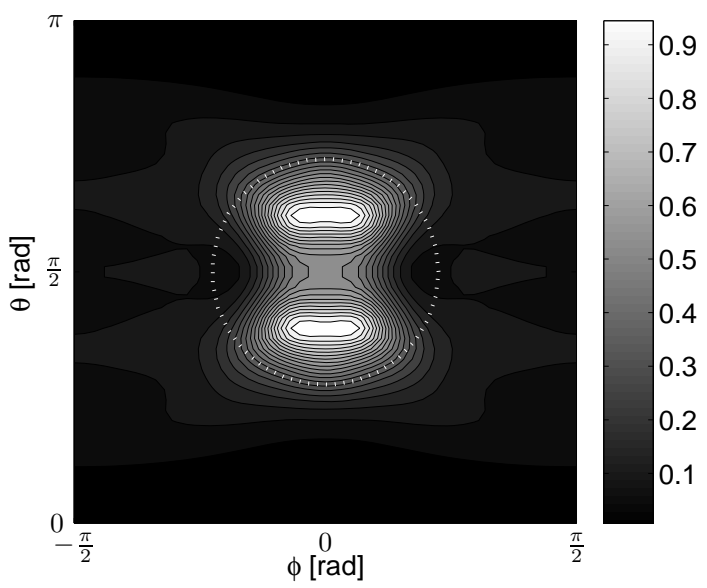

(h) 2.36

Figure 3: (cont.) Angular emission pattern of a single $y$-oriented dipole at the center of the central hole in a $9 \times 9$ hole array. (a) Definition of $\theta$ and $\phi$. (b) $-($ h) Patterns were calculated at a frequency of $375 \mathrm{THz}(800 \mathrm{~nm})$. Subscripts underneath each figure give the aspect ratio of the hole array. A white dotted circle depicts the collection cone of an air objective with an NA of 0.65. As the aspect ratio increases, an emission lobe develops at the center of the emission pattern. With a further increase of the aspect ratio, two side lobes emerge which are more intense than the center peak. 


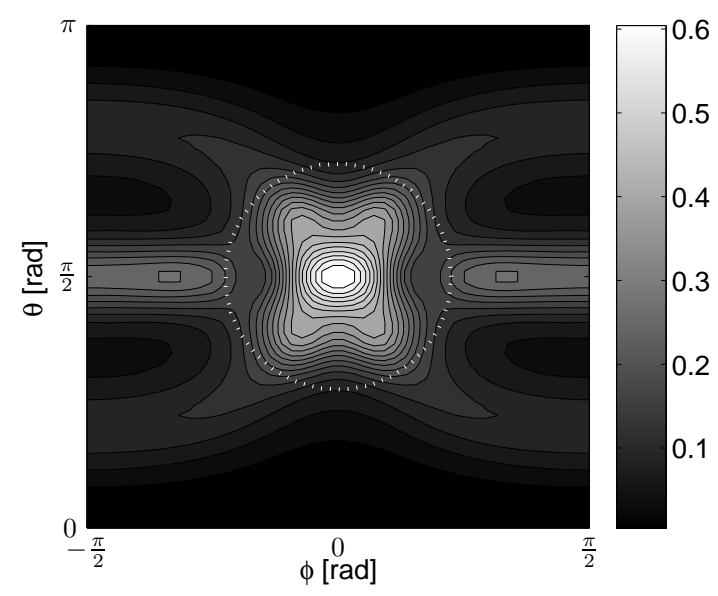

(a) $350 \mathrm{THz}$

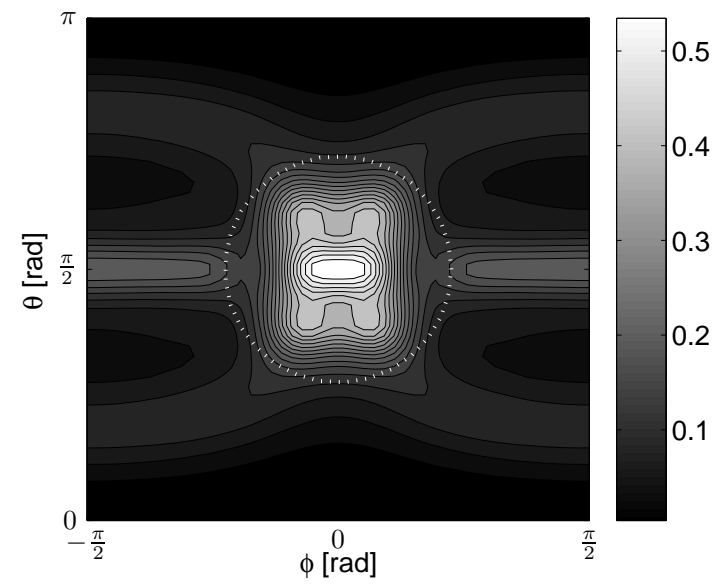

(c) $370 \mathrm{THz}$

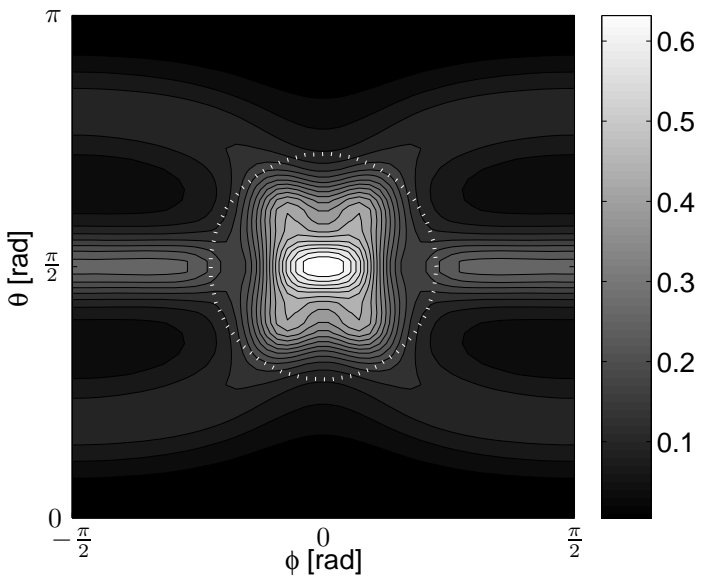

(b) $360 \mathrm{THz}$

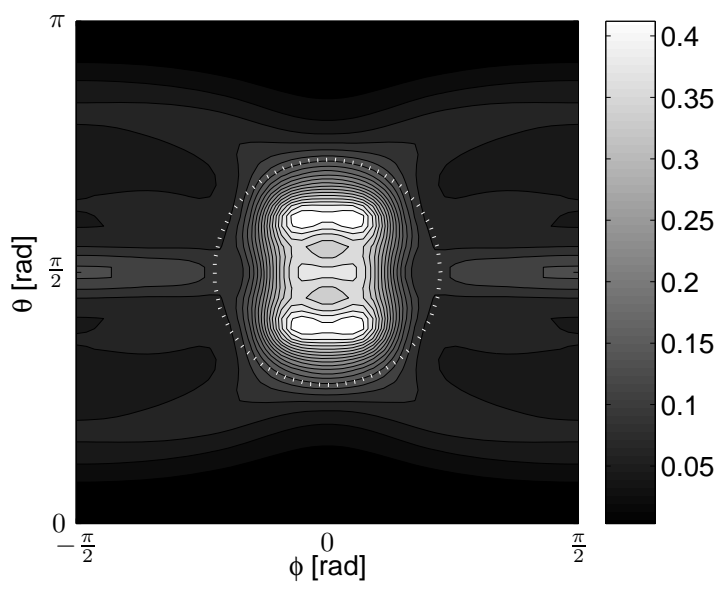

(d) $380 \mathrm{THz}$

Figure 4: Angular emission pattern for a single $y$-oriented dipole in a hole array of $9 \times 9$ holes, as a function of frequency $(350-400 \mathrm{THz})$. The aspect ratio of the holes was fixed to 1.65. As the frequency of emission increases, the magnitude of the center lobe drops and two side lobes emerge. 


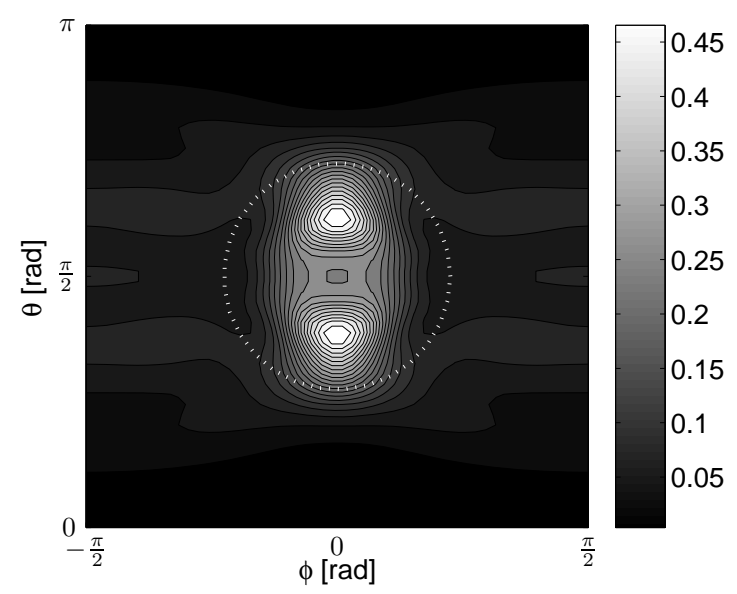

(e) $390 \mathrm{THz}$

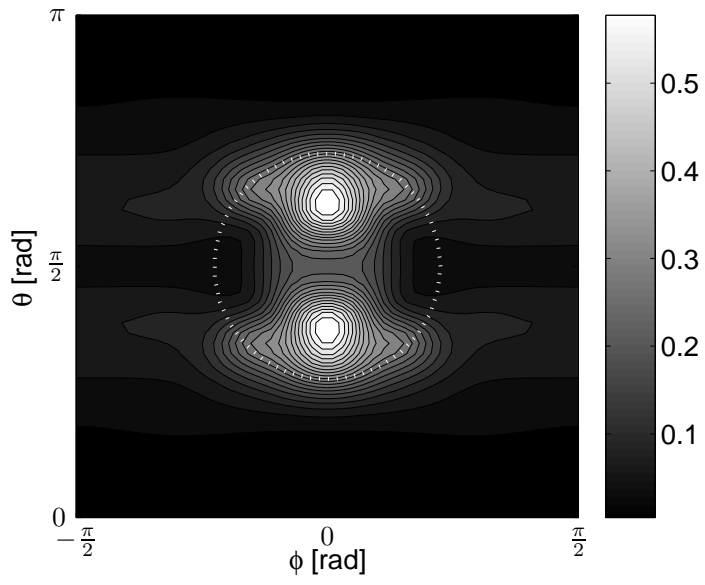

(f) $400 \mathrm{THz}$

Figure 4: (cont.) Angular emission pattern for a single $y$-oriented dipole in a hole array of $9 \times 9$ holes, as a function of frequency $(350-400 \mathrm{THz})$. The aspect ratio of the holes was fixed to 1.65. As the frequency of emission increases, the magnitude of the center lobe drops and two side lobes emerge.

may couple to radiative modes themselves - can improve the apparent quantum efficiency of these emitters. Furthermore, hole arrays are an efficient nanoscale solution to redirect radiation into a desired direction, for example for frequency sorting of emission. ${ }^{41}$ As shown here, the aspect ratio of the holes is a simple parameter to adjust but has a significant influence on the emission pattern.

\section{ACKNOWLEDGMENTS}

This work was supported by the Academy of Finland and CSC - the IT Center for Science, Ltd.

\section{REFERENCES}

[1] Drexhage, K. H., "Interaction of light with monomolecular dye layers," Prog. Opt. 12, 163 (1974).

[2] Chance, R. R., Prock, A., and Silbey, R., "Lifetime of an excited molecule near a metal mirror: energy transfer in the $\mathrm{Eu}^{3+}$ / silver system," J. Chem. Phys. 60, 2184-2185 (1974).

[3] Fleischmann, M., Hendra, P. J., and McQuillan, A., "Raman-spectra of pyridine adsorbed at a silver electrode," Chem. Phys. Lett. 26(2), 163-166 (1974).

[4] Jeanmaire, D. L. and Vanduyne, R. P., "Surface raman spectroelectrochemistry .1. heterocyclic, aromatic, and aliphatic-amines adsorbed on anodized silver electrode," J. Electroanalytical Chem. 84(1), 1-20 (1977).

[5] Ambrose, W. P., Goodwin, P. M., Martin, J. C., and Keller, R. A., "Alterations of single-molecule fluorescence lifetimes in near-field optical microscopy," Science 265, 364-367 (1994).

[6] Xie, X. S. and Dunn, R. C., "Probing single-molecule dynamics," Science 265, 361-364 (1994).

[7] Bian, R. X., Dunn, R. C., Xie, X. S., and Leung, P. T., "Single molecule emission characteristics in near-field microscopy," Phys. Rev. Lett. 75, 4772-4775 (Dec 1995).

[8] Girard, C., Martin, O. J. F., and Dereux, A., "Molecular lifetime changes induced by nanometer scale optical fields," Phys. Rev. Lett. 75, 3098-3101 (Oct 1995).

[9] Trautman, J. K. and Macklin, J. J., "Time-resolved spectroscopy of single molecules using near-field and far-field optics," Chem. Phys. 205, 221-229 (1996).

[10] Novotny, L., "Single molecule fluorescence in inhomogeneous environments," Appl. Phys. Lett. 69, 38063808 (1996). 
[11] Kneipp, K., Wang, Y., Kneipp, H., Perelman, L. T., Itzkan, I., Dasari, R. R., and Feld, M. S., "Single molecule detection using surface-enhanced raman scattering (sers)," Phys. Rev. Lett. 78, 1667-1670 (Mar 1997).

[12] Nie, S. and Emory, S. R., "Probing single molecules and single nanoparticles by surface-enhanced raman scattering," Science 275(5303), 1102-1106 (1997).

[13] Kühn, S., Håkanson, U., Rogobete, L., and Sandoghdar, V., "Enhancement of single-molecule fluorescence using a gold nanoparticle as an optical nanoantenna," Phys. Rev. Lett. 97(1), 017402 (2006).

[14] Anger, P., Bharadwaj, P., and Novotny, L., "Enhancement and quenching of single-molecule fluorescence," Phys. Rev. Lett. 96(11), 113002 (2006).

[15] Tam, F., Goodrich, G. P., Johnson, B. R., and Halas, N. J., "Plasmonic enhancement of molecular fluorescence," Nano Lett. 7(2), 496-501 (2007).

[16] Mertens, H., Biteen, J., Atwater, H., and Polman, A., "Polarization-selective plasmon-enhanced silicon quantum-dot luminescence," Nano Lett. 6(11), 2622-2625 (2006).

[17] Mertens, H., Koenderink, A. F., and Polman, A., "Plasmon-enhanced luminescence near noble-metal nanospheres: Comparison of exact theory and an improved Gersten and Nitzan model," Phys. Rev. B 76(11), 115123 (2007).

[18] Mertens, H., Controlling Plasmon-Enhanced Luminescence, PhD thesis, University of Utrecht (2007).

[19] Ebbesen, T. W., Lezec, H. J., Ghaemi, H. F., Thio, T., and Wolff, P. A., "Extraordinary optical transmission through sub-wavelength hole arrays," Nature 391(6668), 667-669 (1998).

[20] Klein Koerkamp, K. J., Enoch, S., Segerink, F. B., van Hulst, N. F., and Kuipers, L., "Strong influence of hole shape on extraordinary transmission through periodic arrays of subwavelength holes," Phys. Rev. Lett. 92(18), 183901 (2004).

[21] van der Molen, K. L., Segerink, F. B., van Hulst, N. F., and Kuipers, L., "Influence of hole size on the extraordinary transmission through subwavelength hole arrays," Appl. Phys. Lett. 85(19), 4316-4318 (2004).

[22] van der Molen, K. L., Klein Koerkamp, K. J., Enoch, S., Segerink, F. B., van Hulst, N. F., and Kuipers, L., "Role of shape and localized resonances in extraordinary transmission through periodic arrays of subwavelength holes: Experiment and theory," Phys. Rev. B 72(4), 045421 (2005).

[23] Kim, J. H. and Moyer, P. J., "Transmission characteristics of metallic equilateral triangular nanohole arrays," Appl. Phys. Lett. 89(12), 121106 (2006).

[24] van Nieuwstadt, J. A. H., Sandtke, M., Harmsen, R. H., Segerink, F. B., Prangsma, J. C., Enoch, S., and Kuipers, L., "Strong modification of the nonlinear optical response of metallic subwavelength hole arrays," Phys. Rev. Lett. 97(14), 146102 (2006).

[25] Liu, Y. D. and Blair, S., "Fluorescence enhancement from an array of subwavelength metal apertures," Optics Lett. 28(7), 507-509 (2003).

[26] Brolo, A. G., Kwok, S. C., Moffitt, M. G., Gordon, R., Riordon, J., and Kavanagh, K. L., "Enhanced fluorescence from arrays of nanoholes in a gold film," J. Am. Chem. Soc. 127(42), 14936-14941 (2005).

[27] Liu, Y. and Blair, S., "Fluorescence transmission through 1-d and 2-d periodic metal films," Optics Express 12(16), 3686-3693 (2004).

[28] Zhang, J. Y., Ye, Y. H., Wang, X. Y., Rochon, P., and Xiao, M., "Coupling between semiconductor quantum dots and two-dimensional surface plasmons," Phys. Rev. B 72(20), 201306(R) (2005).

[29] Brolo, A. G., Kwok, S. C., Cooper, M. D., Moffitt, M. G., Wang, C. W., Gordon, R., Riordon, J., and Kavanagh, K. L., "Surface plasmon-quantum dot coupling from arrays of nanoholes," J. Phys. Chem. B 110(16), 8307-8313 (2006).

[30] Purcell, E. M., "Spontaneous emission probabilities at radio frequencies," Phys. Rev. 69(11-1), 681 (1946).

[31] Kuhn, H., "Classical aspects of energy transfer in molecular systems," J. Chem. Phys. 53(1), 101-108 (1970).

[32] Lukosz, W. and Kunz, R. E., "Light-emission by magnetic and electric dipoles close to a plane interface. I. Total radiated power," J. Opt. Soc. Am. 67(12), 1607-1615 (1977).

[33] Ford, G. W. and Weber, W. H., "Electromagnetic interactions of molecules with metal surfaces," Physics Reports 113, 195-287 (Nov. 1984). 
[34] Novotny, L., Hecht, B., and Pohl, D. W., "Interference of locally excited surface plasmons," J. Appl. Phys. 81(4), 1798-1806 (1997).

[35] Amos, R. M. and Barnes, W. L., "Modification of the spontaneous emission rate of $\mathrm{Eu}^{3+}$ ions close to a thin metal mirror," Phys. Rev. B 55(11), 7249-7254 (1997).

[36] Hwang, J.-K., Ryu, H.-Y., and Lee, Y.-H., "Spontaneous emission rate of an electric dipole in a general microcavity," Phys. Rev. B 60, 4688-4695 (Aug 1999).

[37] Hermann, C. and Hess, O., "Modified spontaneous-emission rate in an inverted-opal structure with complete photonic bandgap," J. Opt. Soc. Am. B 19(12), 3013-3018 (2002).

[38] Taflove, A. and Hagness, S. C., [Computational Electrodynamics: the finite-difference time-domain method,], Artech House, Norwood, MA, 2 ed. (2000).

[39] Lezec, H. J. and Thio, T., "Diffracted evanescent wave model for enhanced and suppressed optical transmission through subwavelength hole arrays," Optics Express 12(16), 3629-3651 (2004).

[40] Berenger, J. P., "A perfectly matched layer for the absorption of electromagnetic-waves," J. Computational Phys. 114(2), 185-200 (1994).

[41] Aouani, H., Mahboub, O., Devaux, E., Rigneault, H., Ebbesen, T. W., and Wenger, J., "Plasmonic Antennas for Directional Sorting of Fluorescence emission," Nano Lett. 11(6), 2400-2406 (2011). 